\title{
A Case of Infective Endocarditis caused by Salmonella paratyphi A
}

\author{
Egodage UK ${ }^{1}$, Dharmarathne $\mathrm{C}^{1}$, Mohideen $\mathrm{MS}^{1}$, Dahanayake $\mathrm{MU}^{2}$, Bodinayake $\mathrm{CK}^{3}$
}

\section{Introduction}

Endocarditis is a rare complication of salmonellosis. The most frequently observed species is Salmonella typhi[1]. Endocarditis caused by Salmonella paratyphi is extremely rare with only few previous cases documented in the medical literature[2-7]. We report a case of endocarditis caused by Salmonella paratyphi A in an otherwise healthy young patient.

Keywords: Salmonella paratyphi A, Infective endocarditis.

\section{Case Report}

A 40-year-old previously healthy lorry driver was admitted to our unit with complaints of high grade fever associated with chills for one week duration. He also had severe headache, dry cough and constipation for last three days. He was treated by a general practitioner with oral antibiotics. On examination his temperature was $101{ }^{\circ} \mathrm{F}$, auscultation of the chest revealed no murmurs, abdominal examination showed mild hepatomegaly but no splenomegaly. No other relevant findings were elicited. His blood investigations were as follows, hemoglobin - 9.4 g\%, Total WBC count - 13, 200 with $P-72 \%$ and $L-22 \%$, platelet count $-234,000$ and ESR $-60 \mathrm{~mm} / \mathrm{h}$,CRP 48.5.USS abdomen showed mild hepatomegaly and Trans thoracic echocardiogram showed small vegetations attached to anterior mitral leaflet $0.6 \times 0.5 \mathrm{~cm}$.

On the day of admission, three blood culture samples were collected at an interval of half an hour from three different sites and were processed in BACTEC automated blood culture system. His repeated two blood cultures were positive for salmonella paratyphi $A$ after 7 day incubation. It was sensitive for Ampicillin and Ceftriaxone. A trans-oesophageal echo showed multiple ruptured chordae attached to anterior mitral valve with definite vegetations. The patient was started on Ceftriaxone $3 \mathrm{~g}$ daily regimen and the fever and inflammatory markers responded. His temperature came down on initiation of the therapy and he was completely afebrile after 1 day. The diagnosis of Paratyphi A endocarditis is confirmed by positive echocardiogram, blood cultures and by

\footnotetext{
${ }^{1}$ Registrar in Medicine

${ }^{2}$ Senior Registrar in Medicine

${ }^{3}$ Consultant Physician, University Medical Unit, Teaching Hospital, Karapitiya, Galle.
}

positive sero-conversion of $1 / 80$ to $1 / 6404$ weeks apart. Rest of the hospital stay was uneventful and the blood counts and ESR came down gradually. The patient was discharged after one month of admission and he was doing well on review after one month.

\section{Discussion}

Enteric fever is still an important public health problem in many developing countries including Sri Lanka. It is difficult to estimate the real impact of this disease as the clinical symptoms may be confused with other febrile illnesses and specific laboratory confirmation may not be available in most of the time. Enteric fever is a multi-system disease characterized by prolonged fever, sustained blood stream infection, activation of the endothelial system, metastatic infections and immunologic complications due to immune complex deposition leading to multi-organ dysfunction [1]. Overall, enteric fever is caused by Salmonella enterica serovar Typhi (S. Typhi) and Salmonella enterica serovar Paratyphi A (S. Paratyphi A), B and C. However, before the 1990s, S. Typhi was the main causative agent of enteric fever in Southeast Asia and in China, but in the mid-1990s, the number of cases caused by S. Paratyphi A started to increase, and paratyphoid fever subsequently became the major enteric fever [8]. There are only few cases of endocarditis caused by salmonella paratyphi reported in the literature[2-6].This report highlights the importance of considering even the rare cardiac complications of paratyphoid infection when treating patient in order to minimize the disease related morbidity and mortality.

\section{References}

1. Näsström E, Vu Thieu NT, Dongol S et al. Salmonella Typhi and Salmonella Paratyphi A elaborate distinct systemic metabolite signatures during enteric fever. ELife (2014) 5:3.10.

2. Johnson DH, Rosenthal A, Nadas A. Bacterial endocarditis in children under two years of age. American Journal of Diseases of Children,1975;129;183-6

3. Gupta S, Singh B, Kapoor H, Minocha SK, Jain AK.Ventricular septal defect with infective endocarditis caused by Salmonella paratyphi A. Tropical doctor,1994;24;40 
Case Reports

4. Panharoen C, Thisyakorn C, Thisyakorn U, Endocarditis and pericarditis caused by Salmonella paratyphi A. South East Asian Journal of Tropical Medicine and Public Health, 2002;33;161-3.

5. Vaisbein E, Melamed-Snapiri Y, Nassar F, Salmonella paratyphi endocarditis. Acta Cardiology 2006;61 :191-2

6. Subasinghe $\mathrm{S}$, Indrakumar $\mathrm{J}$, An uncommon complication of Salmonella paratyphi A infection. Ceylon Med J, 2010 Jun;55(2): 56-7
7. Sibly A, Tissera $W$,A case of Salmonella paratyphi endocarditis ; a rare complication of paratyphoid fever. Galle Medical Journal, Vol 16: 2011 Sep ;39-40

8. Ochiai RL, Wang X, von Seidlein L, Yang J, Bhutta ZA, et al. Salmonella paratyphi A rates, Asia Emerg Infect Dis 11: 2005;1764-1766 